\title{
Pathophysiology prevention and management of chronic neurogenic constipation in adults
}

\author{
Jacek Hermann" ${ }^{1,}$, Sławomir Michalak ${ }^{2}$, Michał Dopierała ${ }^{1}$, Dariusz Tertoń ${ }^{2}$, Michał Drews ${ }^{1}$ \\ ${ }^{1}$ Chair and Department of General Surgery, Gastroenterological Oncological Surgery and Plastic Surgery, Poznań University of Medical \\ Sciences, Poznań, Poland \\ ${ }^{2}$ Chair and Department of Neurology, Poznań University of Medical Sciences, Poznań, Poland
}

Email address:

jacekhermann@gmail.com(J. Hermann)

\section{To cite this article:}

Jacek Hermann, Sławomir Michalak, Michał Dopierała, Dariusz Tertoń, Michał Drews. Pathophysiology Prevention and Management of Chronic Neurogenic Constipation in Adults. Science Journal of Clinical Medicine. Vol. 2, No. 4, 2013, pp. 122-128.

doi: $10.11648 / j . s j c m .20130204 .11$

\begin{abstract}
Neurogenic constipation occurs most commonly after spinal cord injury, in multiple sclerosis and in Parkinson`s disease. Slow colonic transit time is a major disorder. Patients also develop pelvic floor dysfunction. Spinal cord patients are unable to defecate voluntarily due to the loss of the rectal fullness sensation and they depend on the spinal reflexes. Obstructed defecation in upper motor neuron spinal cord injury and in Parkinson's disease is a result of inability to relax the pelvic floor muscles, whereas in the lower type of injury it is due to hyporeactivity of the rectum. In multiple sclerosis it is a combination of lesions from different levels of the nervous system. In diagnosis, neurological and proctologic examinations are required followed by colonic transit time, defecography and anorectal manometry. Most of the patients respond positively on medical treatment. However, a small group of them requires surgical treatment in the form of either colostomy or ileostomy.
\end{abstract}

Keywords: Neurogenic Constipation, Pathophysiology, Prevention, Management

\section{Introduction}

Constipation belongs to the most troublesome accompanying symptoms, which constitute complaints of patients with severe neurological disorders. Although, constipation is not a life threatening condition it additionally deteriorates their already experienced quality of life. Constipation is diagnosed due to rare evacuations, when faeces are evacuated less frequently than three times a week. Apart from rare defecation, the patients report other more subjective complaints, such as prolonged time of evacuation, hard stools or stools of low volume, sensation of incomplete evacuation, difficulty in expelling of even a soft stool and painful defecation (1). Neurogenic constipation develops in a secondary manner, most frequently following injury to the spinal cord, in multiple sclerosis and in the course of Parkinson's disease. They appear as a result of decreased motor activity of the colon, pelvic floor dysfunction or the loss of the rectal fullness sensation. Immobilization, medications, such as, opioids, anticholinergics, medications administered in Parkinson's disease or factors linked to a diet represent additional variables which may aggravate problems with evacuation of faeces. This study aims at recalling the complex pathophysiology of neurogenic constipation, description of respective preventive approaches and, once they appear, methods of the medical and surgical management.

\section{Innervation and Motor Activity of the Large Bowel}

Comprehension of the large bowel function, including its innervation and the mechanism of defecation is indispensable for understanding of complex neurogenic constipation pathophysiology. Intestinal nervous system plays a key role, to a significant extent independent of absorption, secretion and motor control of the digestive tract and, therefore, it used to be termed "the intestinal brain". It consists of numerous sensory, connecting and motor cells, including the myenteric Auerbach's plexus, located between two layers of muscularis and the submucosal plexus of Meissner. The bipolar fibers of Meissner's plexus, linked at one end to motor neurons of the myenteric plexus and at the other end to the sensory 
fibers running from the receptors of mucosa and the muscularis, represent the centripetal arm of the reflex arch. In turn, the fibers of the myenteric plexus are positioned at the end of the centripetal arm in the reflex arch and they release directly functional potential of myocytes responsible for contractile action of the bowel (2).

A normal motor activity of the large bowel involves a coordinated manifestation of a few types of contractions such as, phasic contractions of a few tens of seconds of the longitudinal muscles, groupped in bands, cause haustration, which do not pass along the intestinal wall. The tonic segmental contractions, which move intestinal contents in the directions opposite to the site of contraction, can be registered within the bowel segments, mainly in the right half of the colon. Peristaltic contractions, on the other hand, transfer the intestinal contents distally. The aforementioned slow types of contractions are responsible, first of all, for accurate mixing, condensation of the contents and formation of the faecal mass. Strong tonic mass contractions, due to the gastro-colic reflex, appear on long segments and transfer faeces toward the rectum and are responsible for initiation of the defecation act. They appear few times a day in the morning hours and following every meal, particularly in the left half of the colon. Normal colonic transport takes 12 through 30 hours from the ileocecal valve to the rectum (3).

As already mentioned above, intestinal nervous system controls involuntary function of the digestive tract. Nevertheless, it remains under control of the central nervous system, somatic and autonomic peripheral innervation, hormonal and humoral factors and of the intestinal contents. As compared to influences of sympathetic system, effects of parasympathetic system prevail (2).

The sympathetic innervation of the colon, up to its splenic flexure, originates from centres located in the lateral horns of the grey matter in the six lower segments of the spinal cord in its thoracic part. The preganglionic fibers run within the white rami communicantes to the sympathetic trunk, to reach sympathetic plexus within the thoracic visceral nerves and, then, enter the superior mesenteric plexus, in which they pass into postganglionic fibers and reach the colon in companion of respective blood vessels. In turn, the sympathetic innervation between the splenic flexure of the colon and upper rectum begins in three proximal segments of lumbar spinal cord. Within lumbar splanchnic nerves, the preganglionic fibres reach intermesenteric and upper hypogastric plexuses, in the inferior mesenteric and superior rectal plexuses they pass into postganglionic fibers and reach the intestinal wall. The superior hypogastric plexus divides into hypogastric nerves, which enter the pelvic plexus. Innervation of the remaining parts of the rectum and the anal canal begins in coeliac plexus and lumbar splanchnic nerves. The nerve fibers form the pelvic plexus, in which pregangionic fibres pass into postganglionic ones and those within the middle and inferior rectal plexuses reach the rectum. Stimulation of the sympathetic nervous system results in inhibited function of the large bowel muscularis and in a stimulated function of the internal anal sphincter (4).

Parasympathetic innervation of the right half of the colon originates from the vagus nerve while the left half of the colon and the rectum is supplied by the parasympathetic sacral nucleus in the S-2 to S-5 segments of the spinal cord. Preganglionic fibers form pelvic splanchnic nerves, which enter the pelvic plexus, become postganglionic fibers and as such they reach the intestine joining intramural fibers and ganglions of intestinal nervous system, participating in formation of the rectal plexuses. Portion of the preganglionic fibers do not pass into postganglionic ones until they reach intramural plexuses. Stimulation of the parasympathetic system increases peristalsis of the large bowel and at the same time cause relaxation of the internal anal sphincter $(4,5)$.

There are some endocrine factors as well as components of the bowel content which stimulate motor function of the bowel. For instance, hormones released from the suprarenal gland cortex decrease the large bowel contractility due to increase water and electrolyte absorption from lumen. In turn, serotonin, prostaglandin E, gastrin, cholecystokinin and motylin act contrarily. Finally, colonic motility can be activated by components of bile due to stimulation of water and electrolyte excretion into the bowel lumen (6).

Striated fibers of the anal sphincter, other muscles composing the pelvic floor and the anoderm are supplied by the motor and sensor fibers of the pudendal nerve and the branches of the sacral plexus originated from segments $\mathrm{S}-2$ through S-4 of the spinal cord (4).

\section{Defecation Act}

Urgency to move bowel initiates the act of defecation as a result of dilatation reflex originating in the neuro-muscle spindles localized within the muscles of the pelvic floor and within the external anal sphincter. Dilation of the rectal wall followed with receptors stimulation is preceded with passage of stool into the rectum due to the peristaltic and mass contractions of the muscular layer within the left half of the colon. The rectum is usually empty except for the defecation act. The recto-sigmoid junction and the resting tone of the muscular layer of the rectum exciding the tone within the sigmoid colon prevent the faeces from entering the rectum. Afterward, the rectal contractions increase pressure in its lumen and the rectal content reaches the upper part of the anal canal initiating the recto-anal inhibitory reflex recognized by Debray. After relaxation of the internal anal sphincter the content of the rectum descents to the upper part of the anal canal and it comes into close contact with the specialized receptors of the anal transition zone which allow discriminating gases from stool. At the same time involuntary constriction of the external anal sphincter, mostly within the lower part of the anal 
canal, prevent the patient from soiling and allows delaying defecation with adaptation of the rectum to a greater volume. The pressure in the lumen of the rectum decreases, urgency lessens and stool or gases return to the sigmoid colon. That involuntary contraction can be prolonged with the voluntary one (7). A defecation reflex which is intramural and spinal as well occurs after voluntary decision to defecate. The former is responsible for increasing peristalsis within the left half of the colon and the rectum and for relaxation of the anal sphincter. The latter strengthens peristalsis of the left colon. The content of the colon enters the rectal ampulla due to the mass contractions. Voluntary relaxation of the external sphincter muscle and of the pelvic floor muscles occurs, including the puborectalis muscle. Then the pelvic floor descends and the ano-rectal angle becomes wide. This is enhanced when thighs are flexed by 90 degrees and on sitting (8). The rectum is a canal for passage of stool rather than a dynamic factor of defecation. Its contractions are too weak for a stool evacuation. Therefore, rise in intra-abdominal pressure due to contraction of the abdominal wall muscles and of the diaphragm is necessary to evacuate faeces. Act of defecation can be interrupted at any moment with voluntary contraction of the external anal sphincter and of the puborectalis muscle. That contraction causes interruption of a faecal column, excretion of the distal part and return of the proximal one to the rectum. Remains of stool retained in the anal canal are subsequently expulsed by alternating peristaltic like contractions of the opposing loops of the external anal sphincter and the act of defecation comes to an end (9).

\section{Spinal Cord Injury}

Spinal cord injury occurs most commonly in young male patients after trauma such as, severe vehicle accidents and fatal water jumping. It produces a syndrome of signs which affect many organs and as a result the life activities of the person (10). Constipation is a clinical manifestation of the large bowel dysfunction. Onset, intensity and clinical course of the symptom depends on the level of injury along the spinal cord. The syndrome is called the upper motor neuron (UMN) bowel, if the lesion is located above the conus medullaris. It is called the lower motor neuron (LMN) bowel if the lesion affects the conus, their axons in the cauda equina, or the pelvic nerves. Although, constipation is one of troublesome accompanying symptoms in the spinal cord patients, loss of voluntary control of defecation with threatening faecal incontinence is the most debilitating symptom. Loss of rectal sensation and of anal discriminatory properties aggravates the risk of incontinence in those patients (11).

Constipation in the patients affected by UMN bowel is a result of impaired colonic motility from the one hand and a type of outlet obstruction from the other. Interruption of inhibitory input from the central nervous system to the intestinal plexus manifests with discoordination of the large bowel contractions in the form of overactive tonic segmental contractions and underactive propulsive peristalsis of the colon. Slow transit constipation is a clinical manifestation of that dysmotility. In turn, outlet obstruction is caused by dyssynergic rise except for physiologic fall of the external anal sphincter activity on straining with faecal retention in the rectum. The striated fibers of the external sphincter and of the puborectalis muscle remain tight as a result of their spastic paresis or paralysis. Spastic contraction of the external sphincter rises additionally in response to the effort of increasing the intraabdominal pressure with contraction of the abdominal wall muscles and the diaphragm (12).

Atrophy of the sacral parasympathetic nucleus occurs with switching off the motor center supplying the muscles of the pelvic floor in the patients with LMN bowel syndrome. The lack of the excitatory influences from the diseased parasympathetic center with prevailing inhibitory stimulation from the sympathetic one results in diminished motility of the colon and in areflexia and atony of the rectum with impending faecal impaction or even ileus. The impaired function of the rectum occurs also due to disruption of the recto-anal inhibitory reflex arch. Faecal incontinence which appears in those patients can be result of either faecal impaction or flaccid paresis or paralysis of the external sphincter with its atrophy and bulging of the perineum (13).

\section{Multiple Sclerosis}

More than half of the patients suffering from multiple sclerosis complain of constipation. Multilevel lesions of the nervous system are responsible for bowel dysfunction in those patients. There is a combination of symptoms and signs resembling both UMN as well as LMN bowel syndromes (14).

\section{Parkinson's Disease}

Constipation in the form of slow transit and outlet obstruction syndrome occur in over half of the patients with severe form of Parkinson's disease. Presumably, dopamine deficiency is responsible for impairment of the colonic motility in those patients. Dopamine is a basic neurotransmitter not only in the central nervous system but also in the enteric nervous one. Increased muscle tone characteristic to the patients with Parkinson's disease affects also muscles of the pelvic floor including the puborectalis as well as the external anal sphincter. That is the cause of a rise except for a fall of the external anal sphincter tone during defecation with faecal retention in the rectum. Constipation is considered by some authors as a risk factor of Parkinson disease. Bowel dysfunction can precede the basic disorder for a few years in part of the patients (15). 


\section{Diagnosis}

It is crucial to collect data from the patient regarding premorbit bowel function such as timing of bowel movement, the volume, consistency, and frequency of stools, and the period of time required for defecation. That information is necessary to prepare the individual bowel management program for the patient the aim of which is restoration of bowel habits present before onset of the disease. Neurological examination enables assessment of the level of injury or lesion within the nervous system.

Proctologic examination provides information about innervation of the pelvic floor muscles including the external anal sphincter and allows for assessment of the rectum regarding possible faecal impaction. The examination reveals the most common anorectal lesions which might be the cause of constipation such as masses, fissures, strictures, and rectocoele. Innervation of the pelvic floor muscles, if only preserved, is examined with digital rectal examination through assessment of the anal tone and spasticity during resting, voluntary contraction of the sphincter, and on straining. The external anal sphincter and puborectalis muscles relaxation occurs on straining with a descent of the pelvic floor by 1 to $3,5 \mathrm{~cm}$. In the spinal cord patients with UMN bowel syndrome and in those with Parkinson disease the anal tone on digital rectal examination is increased with inability to tighten the finger additionally. Apart from that, the anus descends less than 1 $\mathrm{cm}$. In turn, bulging of the perineum below the bone scaffolding with a descent of the anus below tuberosity of the ischium with more than $3,5 \mathrm{~cm}$ appears in the patients with LMN bowel syndrome. In addition, the anus, in those patients, remain open after digital rectal examination with accompanying prolapse of the anal mucosa. Proctologic examination should include assessment of the anocutaneus and the internal anal sphincter reflexes. The former reflex consists of contraction of the external anal sphincter in response to pricking or pinching the anal skin in all four quadrants around the anal verge. Impairment or canceling of that reflex indicates damage to the motor innervation of the sphincter. The latter reflex rely on immediate contraction of the internal anal sphincter after withdrawal of the examining finger. Lack of the reflex may indicate failure of the sympathetic compartment of the rectal autonomic innervation (16).

Comprehensive evaluation of chronic neurogenic constipation requires additional studies such as barium enema and/or endoscopic examination to exclude organic lesions of the large bowel. Those studies are mostly necessary in patients with alarm symptoms and signs such as lower gastrointestinal tract bleeding, "pencil like stools", weight loss, iron-deficiency anaemia, and burden family history. Subsequently colonic transit time, dynamic proctography, and anorectal manometry are recommended.

Colonic transit time is a diagnostic procedure of choice for evaluation of the large bowel motor dysfunction. That procedure is performed with application of radiopaque markers, endoscopic capsules or with scintigraphic techniques. The markers can be available commercially or may be cut from a regular radiopaque nasogastric tube. The patients is given 20 or 24 markers per orally, followed by plain abdominal X-rays taken in 5 and 7 days or after defecation. The test is normal until less than $20 \%$ of markers remain in the bowel and all of them are expelled in 7 days $(17,18)$.

Barium proctography is a dynamic radiological diagnostic procedure indicated in the patients with pelvic floor dysfunction. It allows visualization of an anatomy of the anal canal and the rectum, evaluation of the anorectal angle, and degree of the pelvic floor descent during defecation. The anorectal angle is localized at the intersection of the anal canal axis and a line assigned along the posterior wall of the rectal ampulla. The normal value of the angle is $90^{\circ}-95^{\circ}$ during resting, $125^{\circ}-145^{\circ}$ on straining, and $70^{\circ}-80^{\circ}$ during maximal voluntary contraction. The failure of the anorectal angle to open during defecation can be present in the patients with Parkinson disease as a sign of a pelvic floor dysfunction syndrome. The radiological landmark of the pelvic floor localization is the anorectal junction. The junction during resting is projected on the tuberosity of the ischium or it is located less than $2 \mathrm{~cm}$ underneath the pubococcygeal line. Descending perineum present in the spinal cord patients with LMN bowel syndrome can be recognized if the anorectal junction is found underneath that line of more than $2,5 \mathrm{~cm}$ during resting or of more than $3,5 \mathrm{~cm}$ on defecation (19). Similar evaluation of the anorectal function can be performed with magnetic resonance imaging or ultrasonography programs to avoid radiation (20).

Motor and sensory function of the anal canal and rectum can also be assessed with anorectal manometry. In the spinal cord patients, it is possible to assess whether it is a complete or an incomplete injury because the voluntary anal squeeze pressure is respectively absent or decreased. The recto-anal inhibitory reflex is abolished in the spinal cord patients with LMN bowel syndrome, whereas it is preserved in the patients with UMN syndrome. The patients with bowel dysfunction in the course of Parkinson disease present with a rise instead of a fall of the anal pressure on straining. Finally, the sensory innervation of the anal canal and rectum can be assessed with manometry through evaluation of the lowest volume of air that evokes sensation and a desire to defecate, and the maximum tolerable volume. Assessment of rectal sensation is useful in the spinal cord patients (21).

\section{Medical Treatment}

A bowel rehabilitation program is an individualized plan for the patients with neurogenic bowel dysfunction, the aim of which is effective, efficient, and regular colonic evacuation, prevention of incontinence, and of faecal 
impaction or ileus. The program also involves high fiber diet, fluid balance, increased physical activity if only possible, oral and rectal laxatives, and lavatory equipment $(22,23)$.

Bulking agents increase stool weight due to water retention, decrease colon transit time, and ease defecation (24). Daily fiber requirements range from 20 to 40 gram. Alleviation of constipation can be barely expected in a few weeks after putting a patient on a high fiber diet. A suitable bulking agent should be administered twice a day with a copious amount of fluids. Initially encountered abdominal bloating and colic pains recede in a couple of days. Apart from that, supplementation with proper minerals is necessary because prolonged application of a high fiber diet can lead to iron and calcium deficiency. Bran agents are most of all enriched with fiber exceeding 4 gram in 100 gram of a supplement. The greatest amount of fiber is included in the Ispaghula or Psyllium seed husks (25). If a patient fails to respond to bulking agents, pharmacologic treatment is started. Modification of medications is recommended in a few weeks intervals due to a commonly developed tolerance.

First, osmotic agents such as polyethylene glycol and lactulose among others are administered which act by an osmotic effect. Onset of action occurs in a couple of days. The former, available in sachets containing powder can be administered per orally for months in increasing dosages as much as 30 gram daily. That dosage can be reduced to the least but most efficient amount in a few days (26). The latter composed of galactose and fructose is disposed in a syrup containing 2,5 gram of active substance in $5 \mathrm{ml}$ of solution. The syrup is given from 20 through $40 \mathrm{ml}$ daily depending on the therapeutic effect. Side effects such as meteorism were reported due to a bacterial metabolism of the remedy. Both products may cause transient nausea and vomiting (27).

Next, stimulant laxatives are recommended. They enhance intestinal motility due to stimulation of the myenteric plexus, and alteration of electrolyte and mucous secretion. Bisacodyl with proved efficacy can be administered per orally in the dosage of 5 or $10 \mathrm{mg}$ in 6 through 12 hours before the expected defecation (28). The senna preparations include anthraquinone glycosides and represent another group of commonly used stimulants. Prolonged use of senna products may cause melanosis of the colonic mucosa which is a benign disorder concerning pigmentation of the bowel wall (29). Prokinetic agents such as domperidone and cisapride are recommended in the patients with Parkinson disease in order to increase bowel motility (30).

Since the enteric nervous system is responsible for reflexive motor activity of the large bowel, the spinal cord patients develop spinal automatism of the bowel the result of which are spontaneous evacuations of stools. However, the defecation is unplanned, inefficient, and incomplete. Therefore, the patients may induce efficient and effective evacuation introducing a finger into the anal canal to stimulate the stretch receptors localized within the external anal sphincter and other muscles of the pelvic floor. That stimulation releases preserved reflexes to begin defecation. There is a less efficient intramural reflex in the patients with LMN bowel and more effective reflexes both intramural as well as a spinal one in the patients with UMN bowel. The anal maneuver should be repeated on a regular basis at the same time every day, preferably 30 minutes after a breakfast. The manual stimulation of the rectum should be repeated every second or third day in the patients with UMN bowel. Spastically constricted both external sphincter and the puborectalis muscles should be gently pushed with a finger to relax them, to open the anorectal angle, to facilitate a descent of the pelvic floor, and to start defecation. Ongoing evacuation is accompanied by circular movements of a finger with a gradual dilatation of the anal canal until complete emptying of the rectum. Preserved reflexes are induced with aforementioned maneuvers such as recto-anal reflex with resulting relaxation of the internal anal sphincter and a spinal recto-colonic reflex which cause activation of the large bowel motility. Each finger manipulation should not take more than 1 minute and it should be repeated in 10 minutes intervals until completion of an evacuation. Complete emptying of the bowel manifests with a return of a palpable constriction of the internal sphincter (31).

The spinal cord patients with LMN bowel should empty the large intestine more frequently to prevent them from faecal impaction of the rectum and from an underwear seepage. Tight underclothes and decubitus pillows are recommended them to support descending perineum and to prevent them from prolapse of hemorrhoids and of the rectal mucosa, and to keep the anus close. Evacuation of stools, in those patients, is simply a manual extraction of faeces from the rectum. A finger is introduced into the anal canal to relax a constricted internal sphincter with slow, and round maneuvers. Afterwards, a stool is removed with a bend finger until a return of the sphincter tension. Only a weak intramural reflex from the rectum is released with the manual maneuver in those patients. Therefore, an evacuation should be supported with contraction of the abdominal wall muscles, and the diaphragm, and with a clockwise direction massage of the abdomen, and with a repeated Valsalva maneuver. A lack of regular emptying of the bowel can cause stool impaction of the rectum or ileus (31).

The aforementioned reflexes can be augmented with a topical treatment. Bisacodyl seems to be the most effective stimulant medication. It should be administered in the dosage of $10 \mathrm{mg}$ with a following defecation in 30 to 60 minutes. If patients fail to respond to anal manipulations, and to laxative suppositories, enema is indicated. The enema containing a hypertonic solution of natrium phosphates stimulates excretion of water, and electrolytes to the distal part of the large bowel with a sudden, and watery evacuation of faeces in 2 through 6 hours from the enema application. After developing the spinal automatism, 
topical laxatives should be withdrawn, and an induction of evacuation reduced to the manual, anal maneuvers. Administration of glycerin suppositories makes the withdrawal easier $(28,31)$.

Enema with tap water is recommended in the patients that are mostly in those with LMN bowel, in whom anal manipulations are less effective due to areflexic bowel. Regular enemas provide them with planned and regular evacuations and prevent from constipation and faecal impaction. Tap water in the volume of 250 to $2000 \mathrm{ml}$ is introduced into the rectum through a disposable catheter equipped with a sealing balloon or a regular large bore Foley catheter every day or every second day. However, if faecal impaction occurs, osmotic laxatives together with tap water enemas should be introduced. The agent of choice is polyethylene glycol administered in the dosage of 100 gram per 1 liter of fluid and electrolytes per orally four times a day in a few days. Patients with faecal impaction are put at risk of ileus, ulcerations of the large bowel mucosa, and colon perforation due to the wall ischemia (32).

Biofeedback therapy can be successfully implemented in the management of outlet obstruction syndrome occurring in the patients with Parkinson disease, in spinal cord patients with residual function of the anal sphincter, and in those affected by multiple sclerosis. The aim of that treatment is training of the patients of a coordinated action between muscles of the abdominal wall and of the pelvic floor with a combination of simple exercises. In other words, a voluntary constriction of the abdominal wall muscles, and the diaphragm should occur together with relaxation of the anal sphincter, and the puborectalis muscle during defecation. Effectiveness of biofeedback treatment is estimated on $70 \%(33)$.

\section{Surgical Treatment}

If the patients with neurogenic constipation fail to respond positively on presented medical treatment, and evacuation still is unplanned, inefficient, and incomplete, they are candidates for surgery. Evacuation takes more than an hour in those patients with intractable constipation, they complain of severe headaches and/or abdominal pains during or after defecation, and develop incontinence more frequently than once per month.

Antegrade colonic irrigation through appendicostomy was introduced by Malone in the children treated for faecal incontinence due to spina bifida complications (34). Satisfactory results achieved in children encouraged investigators to implement similar methods in adult spinal cord patients affected by intractable constipation. That procedure allows the patients to empty the bowel completely and on regular basis. Cecostomy can replace appendicostomy in those who underwent appendectomy (35).

There is a small group of spinal cord patients with severe form of neurogenic slow transit constipation who are candidates for either terminal colostomy or ileostomy. Patients with colostomy contrary to ileostomy, frequently require retrograde colonic irrigation to empty the colon satisfactory (36).

Sacral anterior roots stimulation was introduced recently with encouraging results for the treatment of intractable neurogenic constipation, however the method is limited today to clinical trials only (37).

\section{References}

[1] Longstreth GF, Thompson WG, Chey WD, Houghton LA, Mearin F, Spiller RC. Functional bowel disorders. Gastroenterology. 2006;130: 1480-91.

[2] Gabella G. Innervation of the gastroinestinal tract. Int Rev Cytol. 1979; 59: 129-193.

[3] Gill RC, Cote KR, Bowes KL, Kingma YJ. Human colonic smooth muscle: Spontaneous contractile activity and response to stretch. Gut. 1986; 27: 1006-1013.

[4] Woźniak W. Digestive Tract. Human Anatomy. Wrocław: Urban \& Partner; 2001.

[5] Devroede G, Lamarche J. Functional importance of extrinsic parasympathetic innervation to the distal colon and rectum in man. Gastroenterology. 1974; 66(2): 273-80.

[6] Meihoff WE, Kern F Jr. Bile salt malabsorption in regional ileitis, ileal resection and mannitol-induced diarrhea. J Clin Invest. 1968; 47(2): 261-267.

[7] Phillips S.F., Edwards A.W. Some aspects of anal continence and defaecation. Gut. 1965; 6: 396-406.

[8] Henry M.H., Parks A.G. The investigation of anorectal function. Hospital Update. 1980; 1: 29-41.

[9] Shafik A., Ali Y.A., Afifi R. Is the rectum a conduit or storage organ? Int Surg. 1997; 82: 194-197.

[10] Pedersen V, Müller PG, Biering-Sørensen F. Traumatic spinal cord injuries in Greenland 1965-1986. Paraplegia. 1989; 27(5): 345-349.

[11] De Looze D, Van Laere M, De Muynck M, Beke R, Elewaut A. Constipation and other chronic gastrointestinal problems in spinal cord injury patients. Spinal Cord. 1998;36(1): 6366.

[12] Mac Donagh R, Sun W, Smallwood R. Anorectal function in patients with complete supraconal spinal cord lesions. Gut. 1992; 33: 1532-1538.

[13] Devroede G, Lamarche J. Functional importance of extrinsic parasympathetic innervation to the distal colon and rectum in man. Gastroenterology. 1974; 66(2): 273-80.

[14] Hinds JP, Eidelman BH, Wald A. Prevalence of bowel dysfunction in multiple sclerosis. A population survey. Gastroenterology. 1990; 98(6): 1538-42.

[15] Krogh K, Ostergaard K, Sabroe S, Laurberg S. Clinical aspects of bowel symptoms in Parkinson's disease. Acta Neurol Scand. 2008; 117(1): 60-64.

[16] Tantiphlachiva K, Rao P, Attaluri A, Rao SS. Digital rectal 
examination is a useful tool for identifying patients with dyssynergia. Clin Gastroenterol Hepatol. 2010; 8: 955-960.

[17] Hinton JM, Lennard-Jones JE, Young AC. A new method for studying gut transit times using radioopaque markers. Gut. $1969 ; 10: 842-7$

[18] Evans RC, Kamm MA, Hinton JM, Lennard-Jones JE. The normal range and a simple diagram for recording whole gut transit time. Int J Colorectal Dis. 1992; 7: 15-17.

[19] Mahieu P, Pringot J, Bodart P. Defecography: I. Description of a new procedure and results in normal patients. Gastrointest Radiol. 1984; 9: 247-51.

[20] Mahieu P, Pringot J, Bodart P. Defecography: II. Contribution to the diagnosis of defecation disorders. Gastrointest Radiol. 1984; 9: 253-61.

[21] Pucciani F, Ringressi MN. Obstructed defecation: the role of anorectal manometry. Tech Coloproctol. 2012; 16: 67-72.

[22] Astarloa R, Mena MA, Sánchez V, de la Vega L, de Yébenes JG. Clinical and pharmacokinetic effects of a diet rich in insoluble fiber on Parkinson disease. Clin Neuropharmacol. 1992 Oct;15(5):375-80.

[23] Cameron KJ, Nyulasi IB, Collier GR, Brown DJ Assessment of the effect of increased dietary fibre intake on bowel function in patients with spinal cord injury. Spinal Cord. 1996 May;34(5):277-83.

[24] Müller-Lissner SA. Effect of wheat bran on weight of stool and gastrointestinal transit time: a meta analysis. Br Med J. 1988; 296: 615-7.

[25] Sturtzel B, Mikulits C, Gisinger C, Elmadfa I. Use of fiber instead of laxative treatment in a geriatric hospital to improve the wellbeing of seniors. J Nutr Health Aging. 2009; 13: 136-139.

[26] Dipalma JA, Cleveland MV, McGowan J, Herrera JL. A randomized, multicenter, placebo-controlled trial of polyethylene glycol laxative for chronic treatment of chronic constipation. Am J Gastroenterol. 2007; 102: 1436-41.

[27] Tramonte SM, Brand MB, Mulrow CD, Amato MG, O'Keefe ME, Ramirez G. The treatment of chronic constipation in adults. A systematic review. J Gen Intern
Med. 1997; 12: 15-24.

[28] Kienzle-Horn S, Vix JM, Schuijt C, Peil H, Jordan CC, Kamm MA. Comparison of bisacodyl and sodium picosulphate in the treatment of chronic constipation. Curr Med Res Opin. 2007; 23: 691-699.

[29] Marlett JA, Li BU, Patrow CJ, Bass P. Comparative laxation of psyllium with and without senna in an ambulatory constipated population. Am J Gastroenterol. 1987; 82: 333337.

[30] Hiyama T, Yoshihara M, Tanaka S, Haruma K, Chayama K. Effectiveness of prokinetic agents against diseases external to the gastrointestinal tract. J Gastroenterol Hepatol. 2009; 24(4): $537-46$

[31] Clinical practice guidelines: Neurogenic bowel management in adults with spinal cord injury. Spinal Cord Medicine Consortium. J Spinal Cord Med. 1998 Jul;21(3):248-93.

[32] Koch SM, Melenhorst J, van Gemert WG, Baeten CG. Prospective study of colonic irrigation for the treatment of defaecation disorders. Br J Surg. 2008; 95(10): 1273-1279.

[33] Rao SS, Valestin J, Brown CK, Zimmerman B, Schulze K. Long-term efficacy of biofeedback therapy for dyssynergic defecation: randomized controlled trial. Am J Gastroenterol. 2010; 105: 890-896.

[34] Malone PS, Ransley PG, Kiely EM. Preliminary report: the antegrade continence enema. Lancet. 1990;336(8725):12171218.

[35] Worsøe J, Christensen P, Krogh K, Buntzen S, Laurberg S. Long-term results of antegrade colonic enema in adult patients: assessment of functional results. Dis Colon Rectum. 2008;51(10):1523-1528.

[36] Luther SL, Nelson AL, Harrow JJ, Chen F, Goetz LL. A comparison of patient outcomes and quality of life in persons with neurogenic bowel: standard bowel care program vs colostomy. J Spinal Cord Med. 2005; 28(5): 387-393.

[37] Lombardi G, Nelli F, Mencarini M, Del Popolo G. Clinical concomitant benefits on pelvic floor dysfunctions after sacral neuromodulation in patients with incomplete spinal cord injury. Spinal Cord. 2011; 49(5): 629-36. 